\title{
Strong Public Claims May Not Reflect Researchers' Private Convictions
}

\author{
Johnny van Doorn ${ }^{1}$, Don van den Bergh ${ }^{1}$, Fabian Dablander ${ }^{1}$, Noah van Dongen ${ }^{1}$, Koen Derks ${ }^{1}$, \\ Nathan Evans ${ }^{2}$, Quentin Gronau ${ }^{1}$, Julia Haaf ${ }^{1}$, Yoshihiko Kunisato ${ }^{3}$, Alexander Ly ${ }^{1,4}$, Maarten \\ Marsman $^{1}$, Alexandra Sarafoglou ${ }^{1}$, Angelika Stefan ${ }^{1}$, Eric-Jan Wagenmakers ${ }^{1}$ \\ ${ }^{1}$ University of Amsterdam \\ ${ }^{2}$ University of Queensland \\ ${ }^{3}$ Senshu University \\ ${ }^{4}$ Centrum voor Wiskunde \& Informatica
}

How confident are researchers in their own claims? Augustus De Morgan [1] suggested that researchers may initially present their conclusions modestly, but afterwards use them as if they were a "moral certainty". To prevent this from happening, De Morgan proposed that whenever researchers make a claim, they accompany it with a number that reflects their degree of confidence [2]. Current reporting procedures in academia, however, usually present claims without the authors' assessment of confidence.

Here, we report the partial results from an anonymous questionnaire on the concept of evidence that we sent to 162 corresponding authors of research articles and letters published in Nature Human Behaviour (NHB). We opted for NHB because of its broad scope and because the majority of its articles include the main claim in the title (e.g., from the first issue, "Pathogen prevalence is associated with cultural changes in gender equality" [3], or "Attention modulates perception of visual space" [4]), which made it convenient to directly reference the claim in the questionnaire. We selected 129 articles with a claim in the title published between January 2017 and April 2020. The list of selected articles as well as a description of the selection procedure can be found in Appendix A of the online supplement (https://osf.io/zjnpm/). We received 31 complete responses (response rate: 19\%). A complete overview of the questionnaire can be found in online Appendices B, C, and D.

As part of the questionnaire, we asked respondents two questions about the claim in the title of their NHB article: "In your opinion, how plausible was the claim before you saw the data?" and "In your opinion, how plausible was the claim after you saw the data?". Respondents answered by manipulating a sliding bar that ranged from 0 (i.e., "you know the claim is false") to 100 (i.e., "you know the claim is true"), with an initial value of 50 (i.e., "you believe the claim is equally likely to be true or false"). 
Figure 1 shows the responses to both questions. The blue dots quantify the assessment of prior plausibility. The highest prior plausibility is 75 , and the lowest is 20 , indicating that (albeit with the benefit of hindsight) the respondents did not set out to study claims that they believed to be either outlandish or trivial. Compared to the heterogeneity in the topics covered, this range of prior plausibility is relatively narrow.

The lines in Figure 1 connect, for each respondent, their subjective assessment of prior and posterior plausibility; the positive slopes indicate that all 31 respondents believed that the data increased the plausibility of the claim from the title of their article (Wilcoxon signed-rank test, W $=0 ; p<0.001 ; \mathrm{BF}_{-0}=2,670,000$, see https://osf.io/kd4ps/). However, with a median of only 80, the posterior plausibility for their claims is surprisingly low. From the difference between prior and posterior odds we can derive the Bayes factor [5][6], that is, the extent to which the data changed researchers' conviction. The median of this informal Bayes factor is 3 , corresponding to the interpretation that the data are 3 times more likely to have occurred under the hypothesis that the claim is true than under the hypothesis that the claim is false. A Bayes factor of 3 equals Jeffreys's threshold value for labeling the evidence "not worth more than a bare mention" [5], further underscoring the authors' modesty and/or seemingly weak convictions of their article's main claim.

The authors' modesty appears excessive. It is not reflected in the declarative title of their NHB articles, and it could not reasonably have been gleaned from the content of the articles themselves. Perhaps authors grossly overestimated the prior plausibility of their claims (due to hindsight bias); or perhaps they were afraid to come across as overconfident; or perhaps they felt that the title claim was overly general. It is also possible that authors were not sufficiently attuned to the response scale, although none of the respondents indicated that the scales were unclear.

Empirical disciplines do not ask authors to express the confidence in their claims, even though this could be relatively simple. For instance, journals could ask authors to estimate the prior/posterior plausibility, or the probability of a replication yielding a similar result (e.g., (non)significance at the same alpha level and sample size), for each claim or hypothesis under consideration, and present the results on the first page of the article. When an author publishes a strong claim in a top-tier journal such as NHB, one may expect this author to be relatively confident. While the current academic landscape does not allow authors to express their uncertainty publicly, our results suggest that they may well be aware of it. Encouraging authors to express this uncertainty openly may lead to more honest and nuanced scientific communication [7]. 


\section{In your opinion, how plausible was the claim before/after you saw the data?}

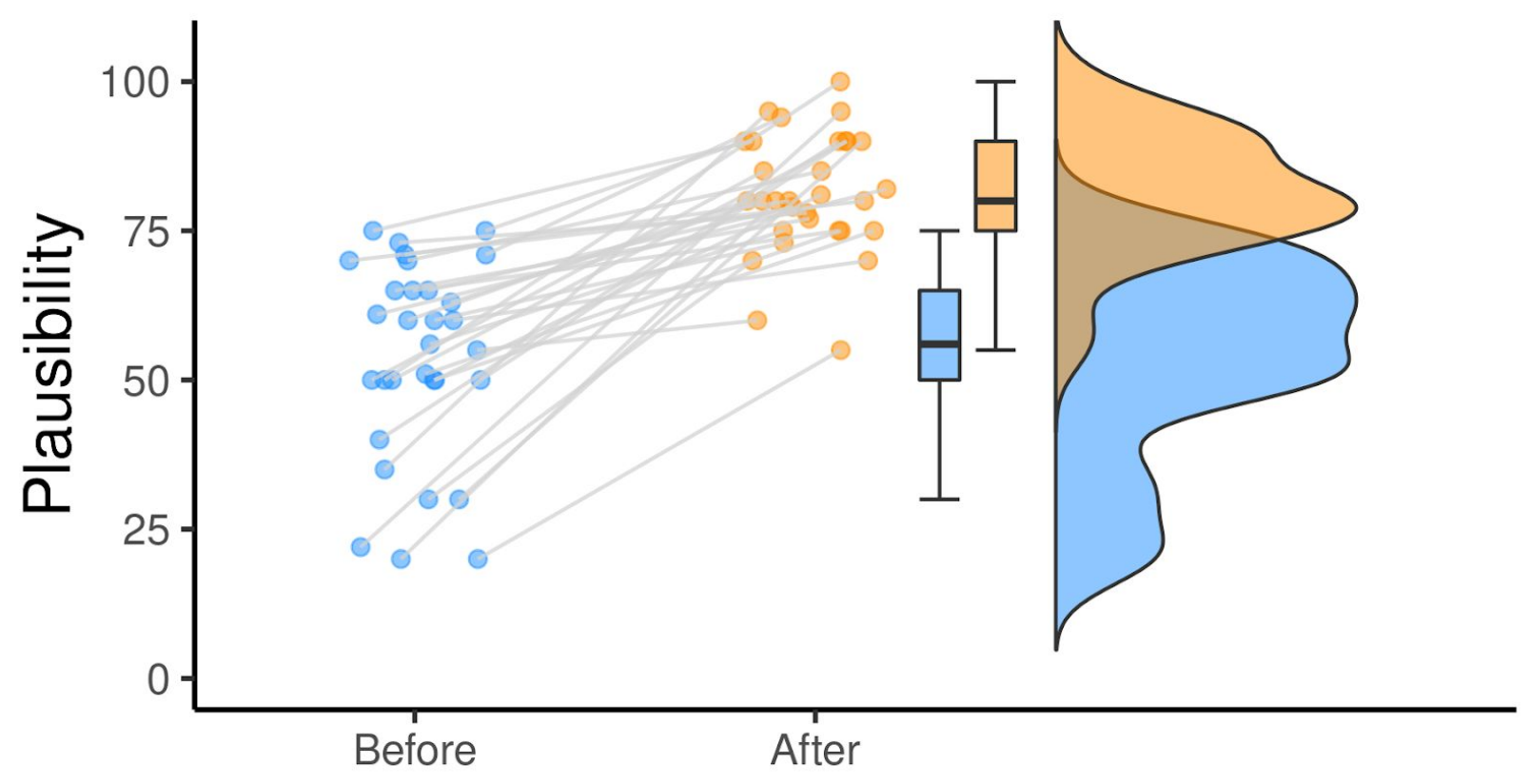

Figure 1: All 31 respondents indicated that the data made the claim in the title of their NHB article more likely than it was before. However, the size of the increase is modest. Before seeing the data, the plausibility centers around 50 (median = 56); after seeing the data, the plausibility centers around 75 (median $=80$ ). The gray lines connect the responses for each respondent. 


\section{References}

[1] De Morgan, A. (1847/2003). Formal Logic: The Calculus of Inference, Necessary and Probable. Honolulu: University Press of the Pacific.

[2] Goodman, S. N. (2018). How sure are you of your result? Put a number on it. Nature, 564, 7.

[3] Zhou, L., Deng, C., Ooi, T. L., \& He, Z. J. (2016). Attention modulates perception of visual space. Nature Human Behaviour, 1, 0004.

[4] Varnum, M. E., \& Grossmann, I. (2016). Pathogen prevalence is associated with cultural changes in gender equality. Nature Human Behaviour, 1, 0003.

[5] Jeffreys, H. (1961). Theory of Probability (3rd ed.). Oxford: Oxford University Press.

[6] Kass, R. E., \& Raftery, A. E. (1995). Bayes factors. Journal of the American Statistical Association, 90, 773-795.

[7] Tell it like it is. Nature Human Behaviour, 4, 1 (2020).

https://www.nature.com/articles/s41562-020-0818-9 